\title{
Safety profile of superior petrosal vein (the vein of Dandy) sacrifice in neurosurgical procedures: a systematic review
}

\author{
*Vinayak Narayan, MD, MCh, Amey R. Savardekar, MD, MCh, Devi Prasad Patra, MD, MCh, \\ Nasser Mohammed, MD, MCh, Jai D. Thakur, MD, Muhammad Riaz, MD, FCPS, and \\ Anil Nanda, MD, MPH
}

Department of Neurosurgery, Louisiana State University Health Sciences Center, Shreveport, Louisiana

\begin{abstract}
OBJECTIVE Walter E. Dandy described for the first time the anatomical course of the superior petrosal vein (SPV) and its significance during surgery for trigeminal neuralgia. The patient's safety after sacrifice of this vein is a challenging question, with conflicting views in current literature. The aim of this systematic review was to analyze the current surgical considerations regarding Dandy's vein, as well as provide a concise review of the complications after its obliteration.

METHODS A systematic review was performed according to Preferred Reporting Items for Systematic Reviews and Meta-Analyses (PRISMA) guidelines. A thorough literature search was conducted on PubMed, Web of Science, and the Cochrane database; articles were selected systematically based on the PRISMA protocol and reviewed completely, and then relevant data were summarized and discussed.
\end{abstract}

RESULTS A total of 35 publications pertaining to the SPV were included and reviewed. Although certain studies report almost negligible complications of SPV sectioning, there are reports demonstrating the deleterious effects of SPV obliteration when achieving adequate exposure in surgical pathologies like trigeminal neuralgia, vestibular schwannoma, and petroclival meningioma. The incidence of complications after SPV sacrifice (32/50 cases in the authors' series) is 2/32 $(6.2 \%)$, and that reported in various case series varies from $0.01 \%$ to $31 \%$. It includes hemorrhagic and nonhemorrhagic venous infarction of the cerebellum, sigmoid thrombosis, cerebellar hemorrhage, midbrain and pontine infarct, intracerebral hematoma, cerebellar and brainstem edema, acute hydrocephalus, peduncular hallucinosis, hearing loss, facial nerve palsy, coma, and even death. In many studies, the difference in incidence of complications between the SPVsacrificed group and the SPV-preserved group was significant.

CONCLUSIONS The preservation of Dandy's vein is a neurosurgical dilemma. Literature review and experiences from large series suggest that obliterating the vein of Dandy while approaching the superior cerebellopontine angle corridor may be associated with negligible complications. However, the counterview cannot be neglected in light of some series showing an up to $30 \%$ complication rate from SPV sacrifice. This review provides the insight that although the incidence of complications due to SPV obliteration is low, they can happen, and the sequelae might be worse than the natural history of the existing pathology. Therefore, SPV preservation should be attempted to optimize patient outcome.

https://thejns.org/doi/abs/10.3171/2018.4.FOCUS18133

KEYWORDS superior petrosal vein; Dandy's vein; venous complications; retrosigmoid approach; neurosurgery

$\mathrm{W}$ ALTER E. Dandy described the anatomical course of the superior petrosal vein (SPV), its relation to the trigeminal nerve and cerebellum, and its significance during surgery for trigeminal neuralgia (TN), in 1929. ${ }^{5}$ SPV, also termed "the vein of Dandy," is an impor- tant venous drainage system in the posterior cranial fossa because it drains the anterior aspect of the cerebellum and brainstem, and ultimately empties into the superior petrosal sinus (SPS). ${ }^{19}$ Neurosurgeons commonly sacrifice this vein to widen the operative exposure at the apex of the cer-

ABBREVIATIONS CPA = cerebellopontine angle; $I A M=$ internal acoustic meatus; $M V D=$ microvascular decompression; $P A M=$ petrous apex meningioma; $P C M=$ petroclival meningioma; PRISMA = Preferred Reporting Items for Systematic Reviews and Meta-Analyses; $S P S=$ superior petrosal sinus; SPV = superior petrosal vein; TN = trigeminal neuralgia.

SUBMITTED March 8, 2018. ACCEPTED April 16, 2018.

INCLUDE WHEN CITING DOI: 10.3171/2018.4.FOCUS18133.

* V.N. and A.R.S. contributed equally to this work and share first authorship. 
ebellopontine angle (CPA) while performing operations for TN, vestibular schwannomas, and petrous or petroclival meningiomas (PCMs). The safety of SPV sacrifice is a challenging question, which has not been addressed adequately in the literature. The aim of our systematic review was to analyze the current surgical considerations of Dandy's vein, as well as provide a concise review of the complications after its obliteration.

\section{Methods}

A systematic review of the literature was performed according to the Preferred Reporting Items for Systematic Reviews and Meta-Analyses (PRISMA) guidelines (Fig. 1). The primary objective of the review was to assess the incidence and nature of the complications associated with sacrifice of SPV. The research question that was primarily addressed was the safety profile after deliberate or inadvertent sacrifice of SPV during surgery. Other related facts pertinent to the topic of interest were also collected, including the applied anatomy and surgical strategies for venous preservation. A detailed search was conducted of electronic databases like PubMed, Web of Science, and the Cochrane database, and this was performed using key MeSH search terms like "superior petrosal sinus," "Dandy's vein," "complications," "sacrifice," "injury," and "retromastoid approach." Given the rarity of definite reporting of the topic in the literature, the search strategy included other synonyms of and terms related to the key search items, with "AND" and "OR" connectors in various combinations to increase its sensitivity.

The primary database search and independent search of the web identified 2335 articles, which was reduced to 1476 articles after removing duplicates. The title review excluded another 1356 articles. Abstracts were reviewed in the next 120 articles, which identified 56 full-text articles related to the topic of interest. Finally, 35 articles were found relevant to our study objectives; these articles were analyzed in detail and the data are presented. As discussed earlier, literature specifically discussing the outcomes after the sacrifice of SPV is scarce and is mostly limited to individual case reports; therefore, estimation of incidence of individual complications was not feasible. Hence, our analysis mostly focused on providing a summary of evidence about venous complications, along with a comprehensive discussion on the feasibility of venous sacrifice during surgery. The data from 3 review articles were not used for the analysis after mutual agreement among the authors. There is no limitation on date or type of publication. We attempt to provide a brief update on the current surgical considerations and a concise review of the expected complications after SPV sacrifice.

\section{Results}

\section{Historical and Anatomical Perspective of Dandy's Vein}

The SPV is the most consistent and prominent vein in the posterior fossa of the embryo, where it is referred to as the "ventral metencephalic vein," and it is the first vein to drain the infratentorial structures in the embryonic period. ${ }^{31}$ Dandy described the SPV as a vein coursing in the
CPA near the rostral aspect of trigeminal nerve. ${ }^{5} \mathrm{He}$ reported the handling of SPV while describing the surgical approach for TN by the cerebellar route in $1932 .{ }^{6}$ Dandy stated, "Electrocautery makes it possible to easily coagulate and divide the petrosal vein should this be necessary. The control of the petrosal vein and its branches was really the only element of danger in the operation and safely overcome, if necessary, with the cautery. As a matter of fact it is only once in about fifteen cases that it is necessary to occlude the petrosal vein for in the remaining cases the sensory root is not at all obscured by the vessel." The interpretation could be that if the vein obscures the surgical view, it can be safely sacrificed; however, this surgical step has to be seen in the light of the era preceding operating microscopes in which Dandy was performing these challenging surgeries..$^{9,22}$

The anatomy of the SPV complex was clarified by Huang et al. in their study on the classification of the posterior fossa venous system. ${ }^{12}$ The tributaries of the SPV together create large infratentorial venous channels termed the "SPV complex." The SPV is the venous structure most frequently encountered during lateral posterior fossa approaches. ${ }^{31}$ The most common tributaries of the SPV complex are the cerebellopontine fissure vein, middle cerebellar peduncle vein, transverse pontine vein, pontotrigeminal vein, and the veins draining the lateral cerebellar hemisphere. These veins merge together along the adjacent anterolateral margin of the cerebellum. ${ }^{19}$ The SPV may be either the terminal segment of a single vein or the common stem arising from a union of several of the aforementioned veins. ${ }^{31}$ Matsushima et al. proposed the classification of SPVs into lateral, intermediate, and medial groups on the basis of the relationship of their site of entry to the internal acoustic meatus (IAM)., ${ }^{9,21}$ The intermediate group drains into the sinus above the IAM, the medial group drains into the sinus medial to the IAM, and the lateral group drains into the sinus lateral to the IAM. ${ }^{11}$

Huang et al. reported that the SPV usually drains into the SPS just posterior to the Meckel cave and rarely superior to the IAM. ${ }^{12}$ However, based on further studies showing that the stem of the SPV complex empties into the SPS, nearer to the Meckel cave than to the IAM, Tanriover and Rhoton's group updated the classification as type 1, type 2 , and type 3 based on the relationship of its site of entry into the SPS, the Meckel cave, and the IAM (Fig. 2). ${ }^{31}$ In type 1, the SPV complex empties into the SPS superior or lateral to the IAM, particularly at a point superolateral to the medial limit of the facial nerve at its entry point to the IAM. In type 2, the SPV complex empties into the SPS between the lateral limit of the trigeminal nerve (at its entry point to the Meckel cave) and the medial limit of the facial nerve (at its entry point to the IAM). In type 3, the SPV complex empties into the SPS at a point medial to the lateral limit of the trigeminal nerve at its entry point into the Meckel cave. Although there are large-diameter anastomotic venous channels directed to the ipsilateral supratentorial deep veins for compensatory venous drainage in SPV occlusion, a similar anastomotic system to the contralateral petrosal venous complex may not provide adequate compensatory outflow. ${ }^{11}$ The intraoperative images of SPV and related structures are given in Fig. 3. 


\section{Web search}

PubMed, Web of Science, Cochrane database

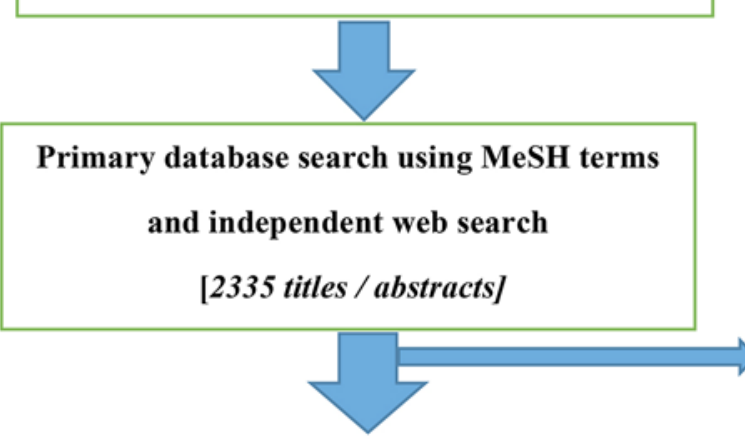

Duplicates are excluded

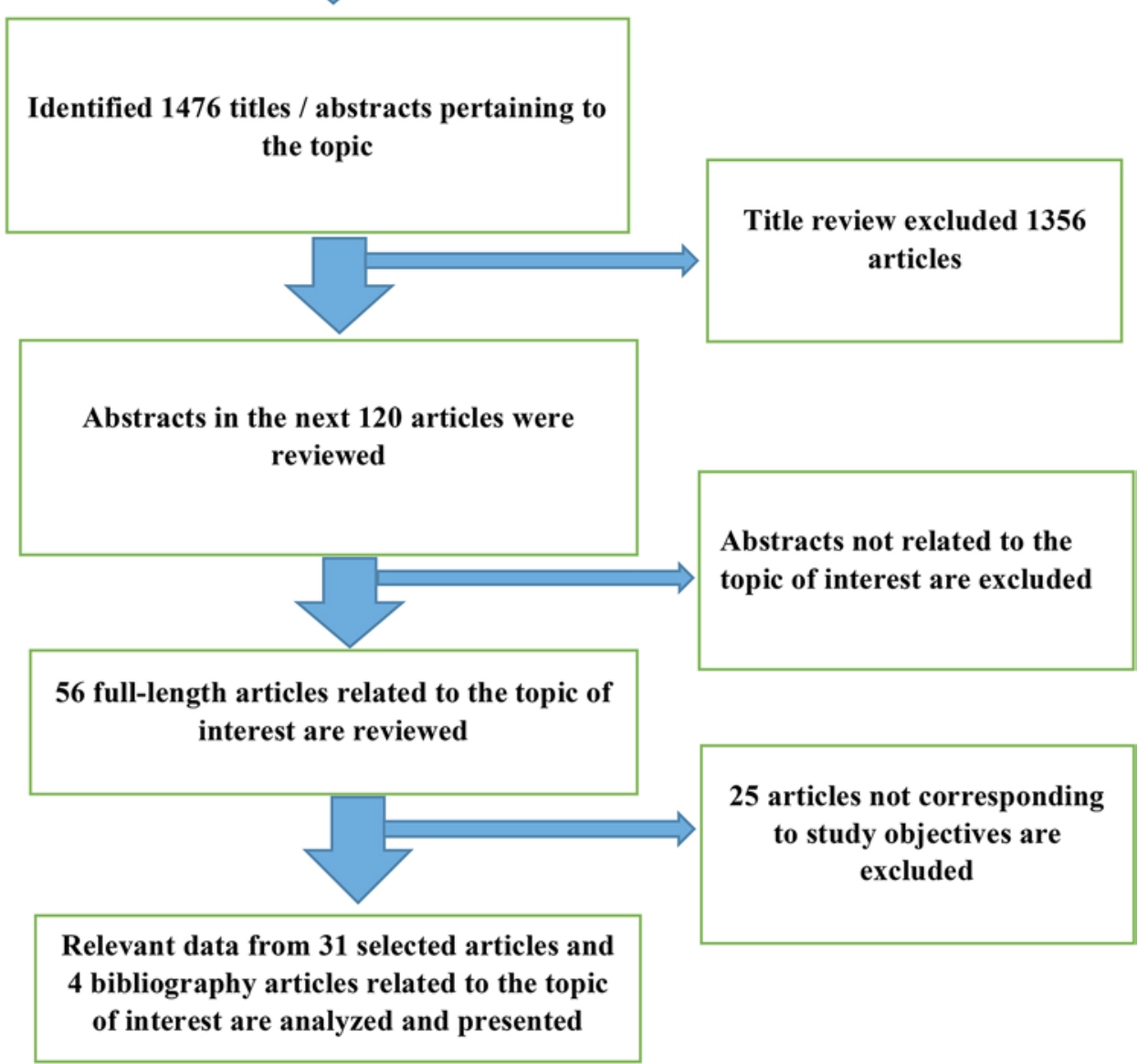

FIG. 1. PRISMA protocol showing the final selection of articles.

\section{Surgical Implications of the Vein of Dandy}

The vein of Dandy is the superior division of the petrosal venous drainage complex, and it is usually a large, multistemmed structure obstructing the approach to the trigeminal nerve from the retrosigmoid corridor. ${ }^{24}$ It acts as a significant anatomical landmark as well as a hindrance for the retrosigmoid approach in that it limits the extent of cerebellar retraction and the visualization of the superior (supratrigeminal) corridor (especially while dealing with PCM). Dealing with the vein of Dandy when it is in the "line of fire"-limiting cerebellar retraction or hindering the key neurosurgical step-is controversial. Most surgeons believe that it is justified to sacrifice this vein at such a juncture; however, there is an evolving counterview (especially in this neurosurgical era, which emphasizes complication avoidance) that SPV preservation is desirable in all cases.

There are studies describing almost negligible effects of SPV sectioning in posterior fossa surgeries. Samii et al. and Mizutani et al. reported negligible effects after sectioning of SPV in their series of many patients with 

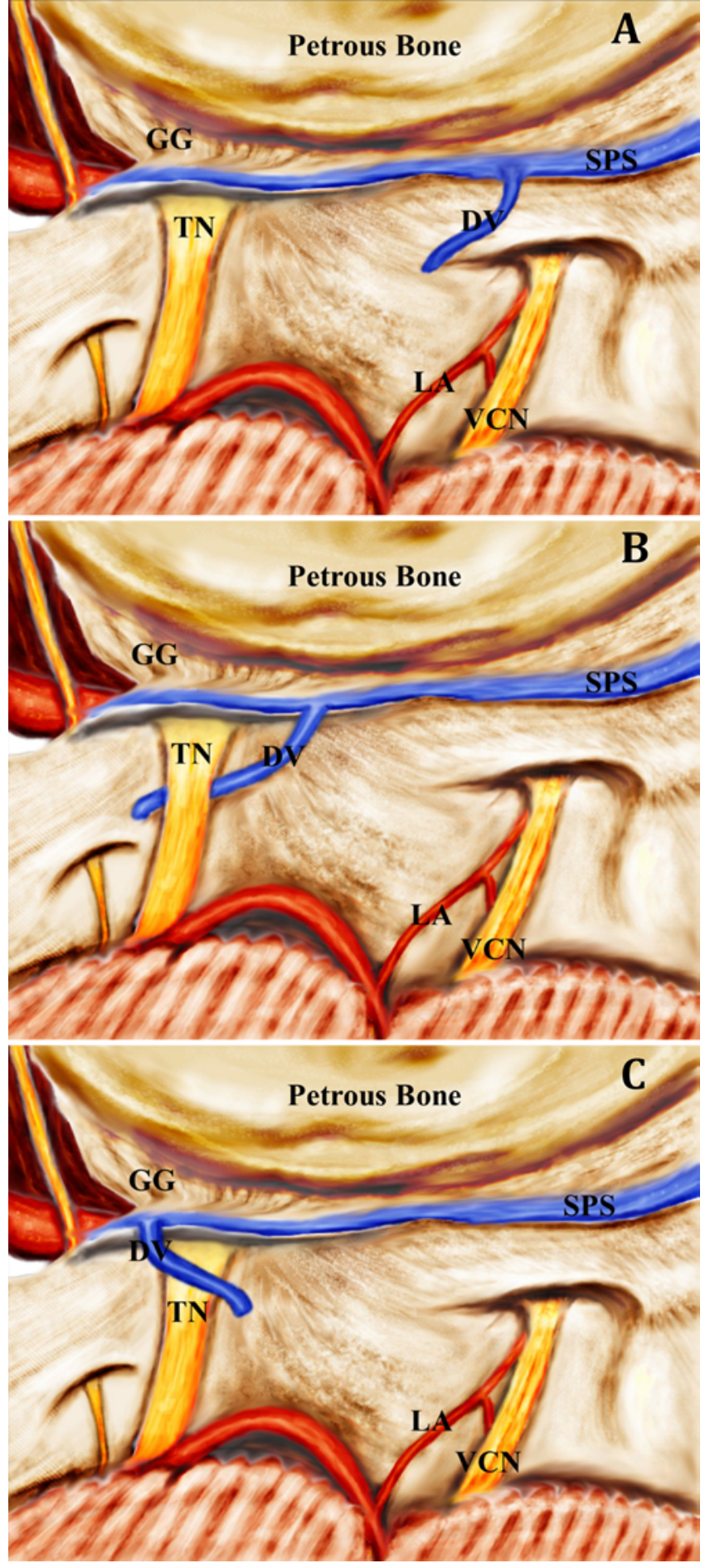

FIG. 2. Illustrative images showing types of SPV complex. A: Type 1 SPV complex. B: Type 2 SPV complex. C: Type 3 SPV complex. DV = Dandy's vein; $G G$ = gasserian ganglion; $L A=$ labyrinthine artery; $T N=$ trigeminal nerve; $\mathrm{VCN}=$ vestibulocochlear nerve.

petrous apex tumors. They hypothesize that because the SPV was significantly displaced or compressed by tumor, the collateral veins must have already developed. ${ }^{23,28}$ McLaughlin et al. described in their extensive series of 4400 microvascular decompression (MVD) surgeries that
SPV can be sacrificed without major morbidity or mortality. ${ }^{22}$ Gharabaghi et al. also reported that the obliteration of petrosal vein during surgery for petrous apex meningioma (PAM) did not have any major influence on postoperative outcome. ${ }^{10}$ In their series of 55 patients with PAM, the vein of Dandy was sacrificed in 27 (49\%) patients and the postoperative complication of hearing loss was noted in 3 (11\%) patients; however, a similar deficit was also noted in $11 \%$ of the cohort in which veins were preserved. Pathmanaban et al. published their experience of 184 patients with coagulation and division of the SPV during MVDs. ${ }^{24}$ The overall rate of venous complications in this study was 2.7\%; however, no case of venous infarction was noted in 184 patients who had obliteration of the SPV. The study also reported that the incidence of venous infarction after SPV obliteration in MVD surgeries was < $0.5 \%$. Elhammady and Heros mention sacrificing the SPV while performing MVD surgery in their large cohort of patients with TN, and do not attribute any morbidity directly to it. ${ }^{9}$ A retrospective review of the senior author's (A.N.) personal series of 50 MVD cases surgically treated for TN in the last 5 years showed SPV sacrifice in 32 cases. The incidence of complications after SPV sacrifice was $6.2 \%$ $(2 / 32)$ compared with $0 \%(0 / 18)$ in the group with preserved SPV. One complication was major (cerebellar and brainstem edema), and the other complication was minor (mild cerebellar edema). The senior author maintains a low threshold for SPV sacrifice at times when it obstructs the key step in the surgical procedure.

At the other end of the spectrum, some reports demonstrate the deleterious effects of SPV obliteration when achieving adequate exposure in surgically treated pathologies like TN, vestibular schwannoma, and PCM. The incidence of complications reported in various case series varies from $0.01 \%$ to $31 \%$, based on our review. Masuoka et al. reported a case of a 77-year-old man who developed cerebellar hemorrhagic venous infarction after MVD surgery for $\mathrm{TN}$, in which the surgeons had sacrificed the common stem of the 3 tributaries of SPV. ${ }^{19}$ Another patient in the same series developed postoperative visual and auditory hallucinations explained by the sectioning of SPV during MVD. Similarly, Inamasu et al. reported the severe complication of intracerebral hematoma following sectioning of SPV in surgery for cystic vestibular schwannoma. ${ }^{13}$ Koerbel et al. also published their complications of venous infarction $(0.3 \%)$ and life-threatening hydrocephalus $(0.03 \%)$ in their series of PAM surgeries after the obliteration of the vein of Dandy. ${ }^{15}$ A summary of published reports on the deleterious effects of SPV obliteration is given in Tables 1 and 2.2,3,7,10,13-16,18,19,23-26,29,30,32-34

Myriad complications are associated with obliteration of SPV while operating on posterior fossa pathologies. These complications include hemorrhagic and nonhemorrhagic venous infarction of cerebellum, sigmoid thrombosis, cerebellar hemorrhage, midbrain and pontine infarct, intracerebral hematoma, cerebellar and brainstem edema, acute hydrocephalus, peduncular hallucinosis, hearing loss, facial nerve palsy, coma, and even death. ${ }^{18}$ Most of these complications were managed conservatively; however, reexploration and decompressive surgery was required in a few cases. The delay in neurological recovery 

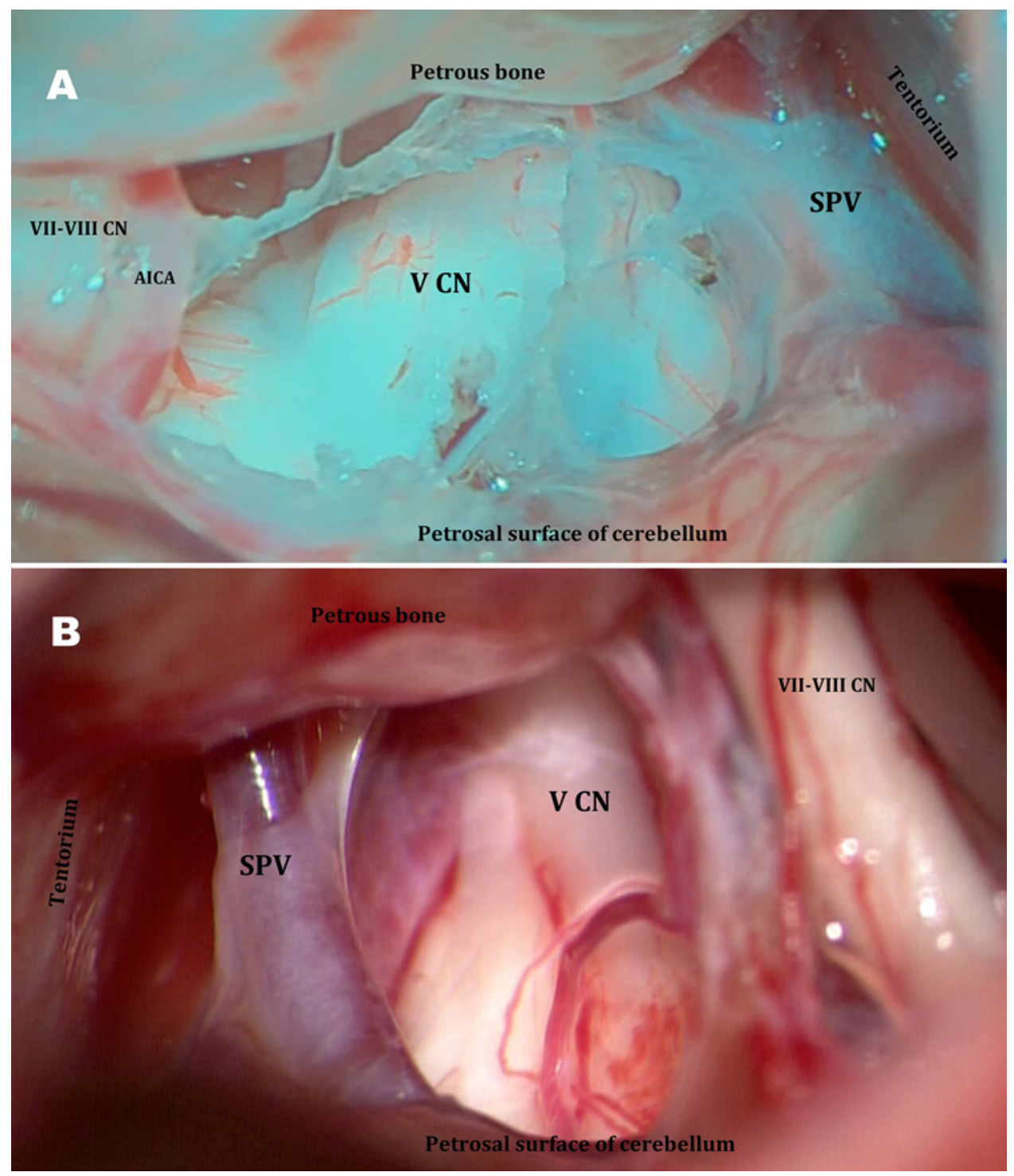

FIG. 3. Intraoperative images of the vein of Dandy showing the anatomical relationship with adjacent structures. AICA = anterior inferior cerebellar artery; $\mathrm{CN}=$ cranial nerve.

after the complications was common in most of the published studies.

Whether venous neurovascular conflicts give rise to TN is a matter of debate, but TN often involves the SPV system, and this forms an important issue in our discussion-when the SPV itself is the offending agent, how do neurosurgeons resolve the neurovascular conflict? ${ }^{7,8} \mathrm{Kuncz}$ et al., in their series of 287 consecutive patients with TN, operated on 103 MR angiography-positive cases (i.e., demonstrating vascular conflict). ${ }^{17}$ At surgery, pure venous compression was found frequently $(31.2 \%)$ in the atypical TN symptomatology group ( $\mathrm{n}=17$ cases) and rarely $(1.2 \%)$ in the typical TN symptomatology group ( $\mathrm{n}=86$ cases). In their series, the veins were divided or sacrificed in most cases of venous neurovascular conflict, to make sure that there was no chance of compression following the MVD. The authors in this series did not report on any postoperative complications. In another large series of 313 patients who underwent operation for TN, Dumot et al. reported that venous neurovascular conflict was the predominant cause in 55 patients $(17.5 \%))^{7}$ The conflicting veins belonged to the superficial SPV system in 36 patients and to the deep SPV system in 19 patients. Even when dealing with the SPV as an offending agent, Dumot et al. recommended avoiding the sacrifice of veins as much as possible. They advised dissecting the root free from all arachnoid filaments and adhesions, starting from the trigeminal root entry zone at the brainstem up to the porus of the Meckel cave. During this maneuver, the compressive veins were dissected free and detached from the trigeminal root. If even this could not achieve effective decompression, then coagulation and division of the vein was considered to relieve the root. These authors reported postoperative complications in the form of cerebellar infarction needing decompression in two patients-one of which was attributed to sacrifice of the pontine afferent branch of the SPV. Considering their 


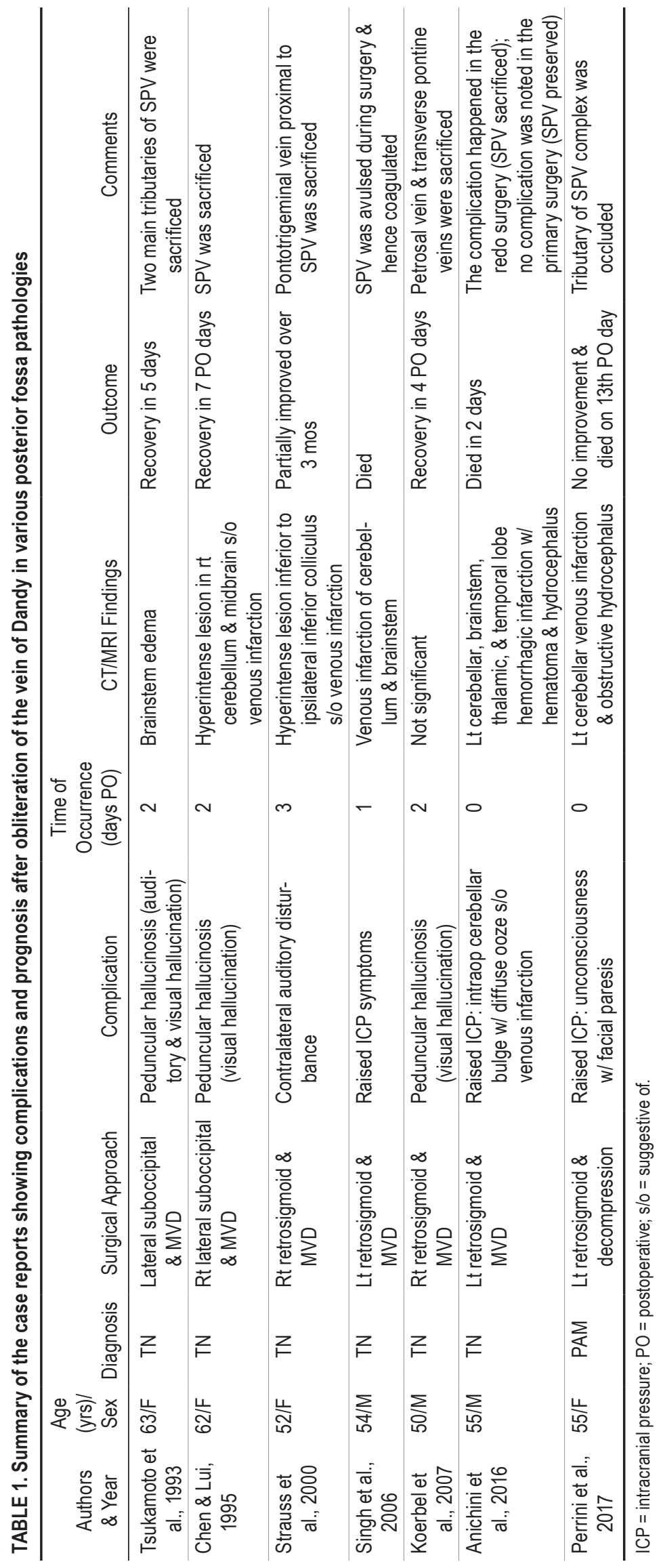




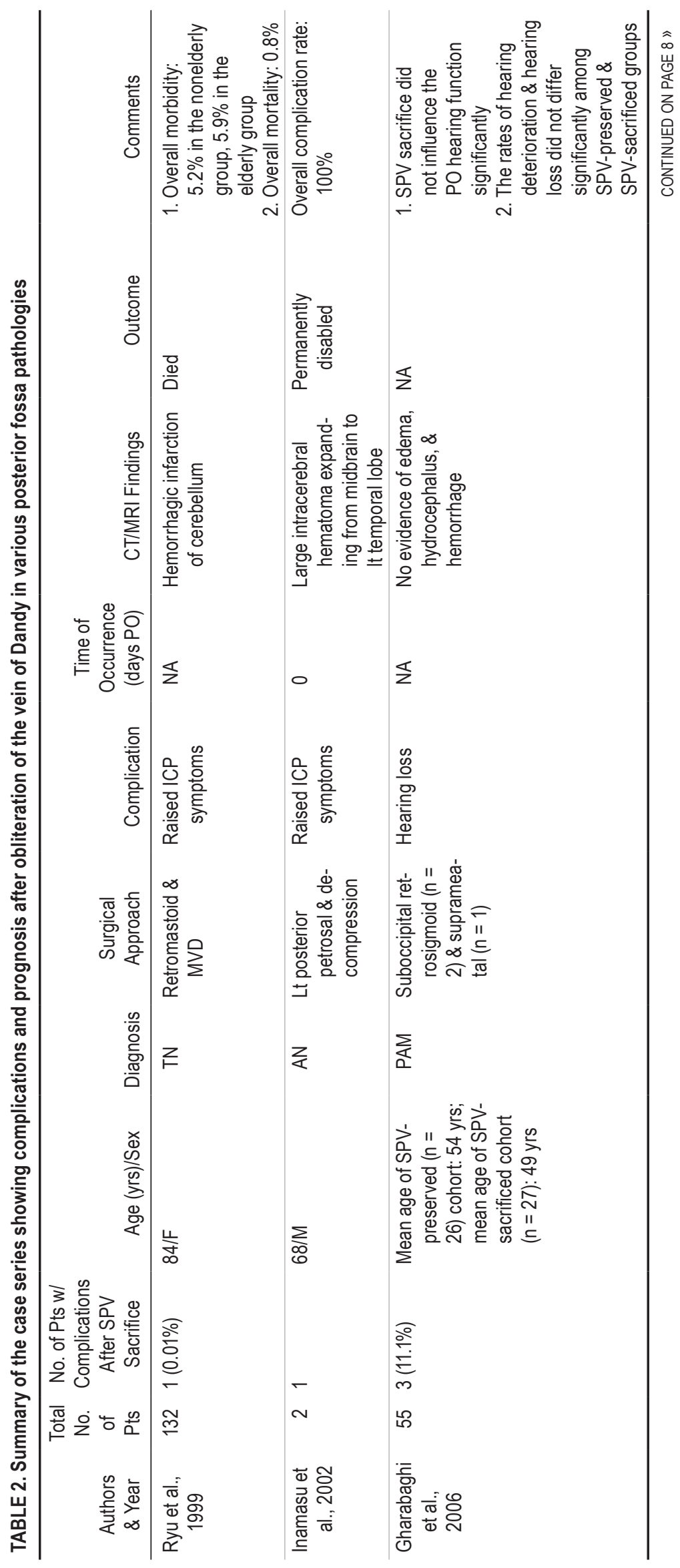

Neurosurg Focus Volume 45 • July 2018 


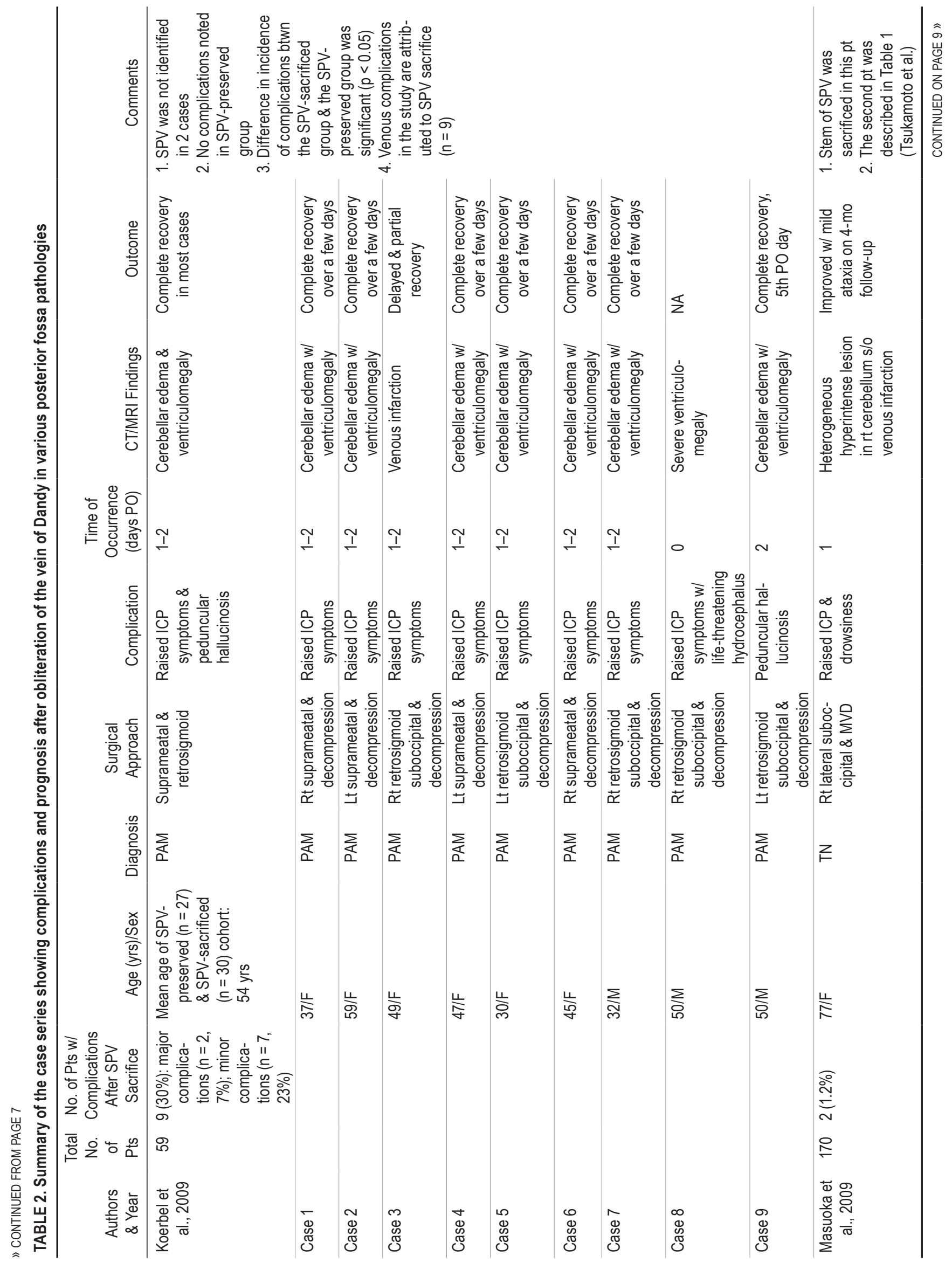




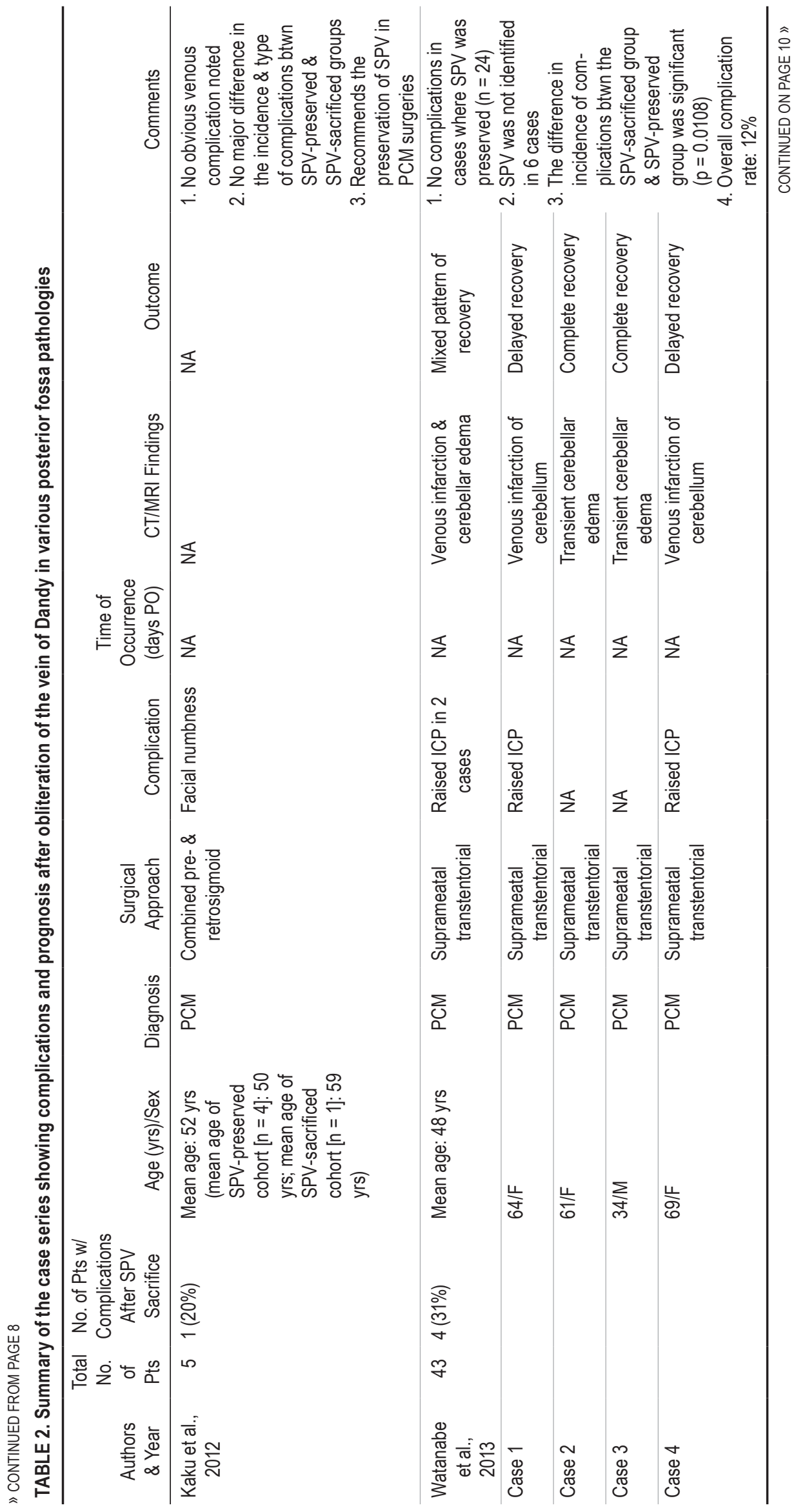

Neurosurg Focus Volume 45• July 2018 


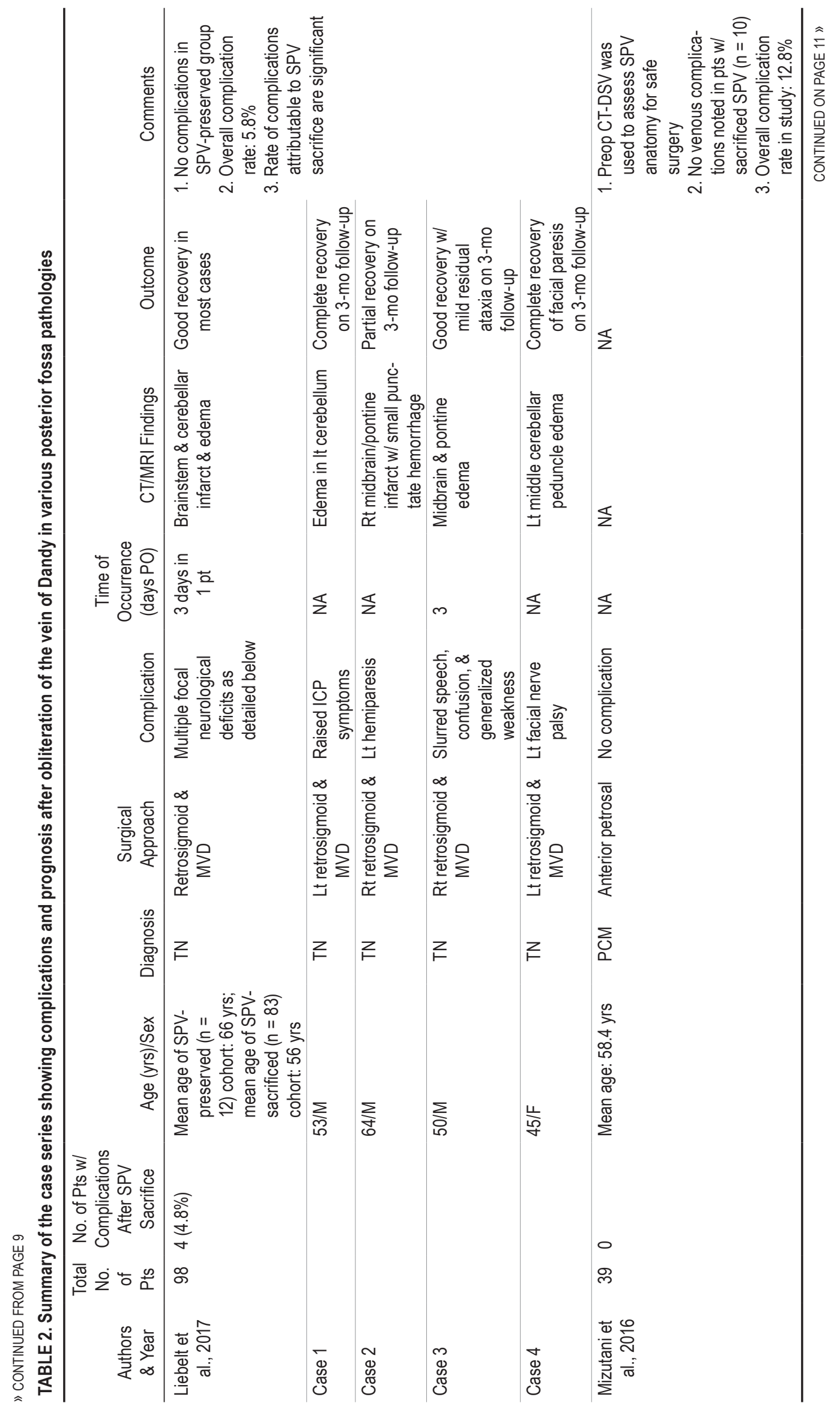




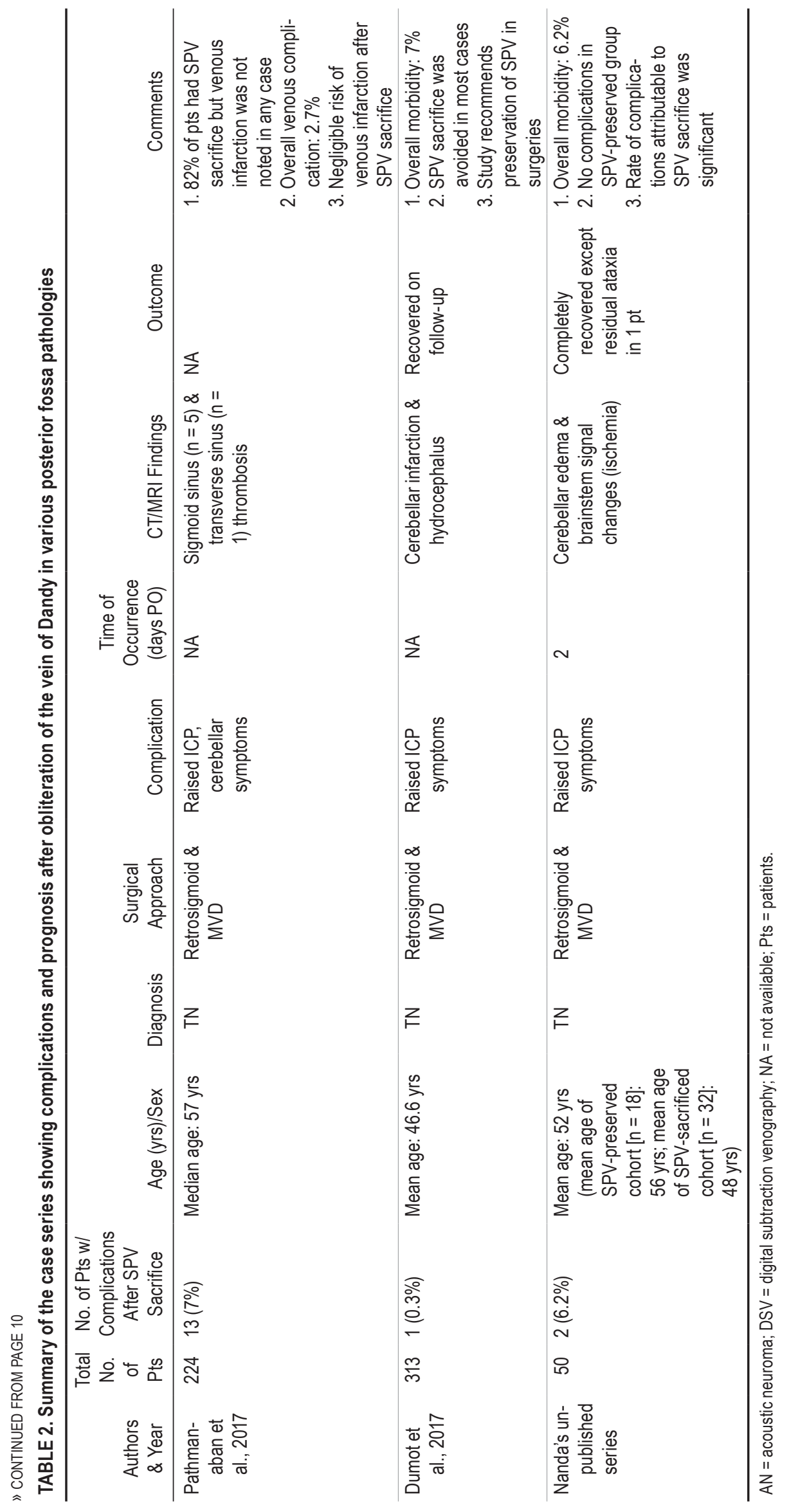

Neurosurg Focus Volume 45 • July 2018 
venous-preserving approach, this complication may have been inevitable; however, at another level, this complication highlights the fact that blatant venous sacrifice may not be justified. Thus, handling of the SPV remains contentious even in this setting.

\section{Discussion}

The knowledge of venous anatomy and its variations is considered essential in devising any strategy in a skull base approach. ${ }^{27}$ The importance of preserving the venous outflow while performing a posterior skull base approach has been emphasized and recognized. ${ }^{1}$ The consensus on the preservation or sacrifice of the SPV during the retrosigmoid and petrous approaches remains a neurosurgical inconsistency, with proponents on either side.

The common neurosurgical conditions in which complications evolved after obliterating SPV are MVD for TN and petrosal approaches for PCM. On many occasions, the common stem of the tributaries of the SPV was sectioned for better visualization of the trigeminal nerve. ${ }^{19}$ Most of the time, the complications occurring after obliteration of the vein of Dandy are underreported due to the transient nature of postoperative deficits as well as to inaccurate interpretation of the postoperative radiology. Cerebellar edema is a common finding in many cases in which complications are attributed to SPV obliteration, and its characteristic features are prominent edema in the deep part of the cerebellum, hemorrhage within the lesion due to venous hypertension or venous infarction, and noncongruency of the lesion in a known arterial territory but compatible with SPV drainage territory.

The incidence of complications after the obliteration of SPV varies. Watanabe et al. and Koerbel et al. reported approximately $30 \%$ venous-related phenomena after the obliteration of SPV in patients with petrous meningioma. ${ }^{15,33}$ Cheng noted hemorrhage as the most common severe complication. ${ }^{4}$ Another study described a rate of $23 \%$ minor complications and $7 \%$ major complications (life-threatening hydrocephalus and cerebellar venous infarction) after severing of the SPV. ${ }^{15}$ Of the 149 patients who underwent operation for acoustic neuroma, Xi et al. reported the preservation of SPV in 141 cases and sacrifice of SPV in 8 cases. ${ }^{34}$ The postoperative complications reported were operative site hematoma $(n=40$ vs $n=4)$, cerebellar edema $(n=56$ vs $n=5)$, and cerebellar hemorrhage $(n=12$ vs $n=3)$ in the SPV-preserved and SPV-sacrificed groups, respectively. The study highlighted the significant difference in the incidence of cerebellar hemorrhage ( $p=0.05$ ) between both groups and recommended SPV preservation to avoid postoperative cerebellar hematoma. The occurrence of complications after sectioning of the vein of Dandy in petrous or petroclival meningioma and MVD surgeries depends on various factors. Even though the preoperative prediction of such complications may not always be possible, various anatomical, radiological, and surgical factors help in predicting the incidence of complications due to SPV sectioning. These factors are anatomical variations of the SPV complex and its tributaries, degree of compensation by anastomotic venous collaterals, size of the SPV, selection of surgical approach, duration and severity of simultaneous cerebellar retraction, extent of arachnoid sleeve dissection over the vein, type of attachment of CPA meningioma, and sectioning of the main stem versus small-diameter tributaries., ${ }^{4,15,29}$

The proper extension of arachnoid sleeve dissection helps in better mobilization of the vein and easy maneuvering to get proper trigeminal nerve exposure. This dissection may suffice to expose the trigeminal nerve, thus avoiding sacrifice. In cases in which sacrifice may be warranted, sectioning of the SPV stem (very close to the confluence of its tributaries) or its major tributary (e.g., cerebellopontine fissure vein, the largest bridging vein in the CPA) may lead to extensive infarction of the petrosal surface of the cerebellum as well as the brainstem and should be avoided..$^{19,21}$ The anastomotic channels of the SPV complex are usually well developed in the ipsilateral side, and so in many cases minor tributaries can be easily sacrificed without major problems (as the major tributaries can take over the drainage), and sectioning of the stem segment would be better avoided., ${ }^{4,7}$ The diameter of the vein is a matter of concern, and a vein with a diameter $<2$ $\mathrm{mm}$ could be coagulated and cut without risk. ${ }^{35}$ Koerbel et al. also mentioned in their study that $78 \%$ of patients with venous complications had obliteration of a large-diameter SPV. ${ }^{15}$ These investigators also suggested that the diameter of the severed SPV ( $\geq 1.3 \mathrm{~mm}$ ) had significant predictive value for the incidence of venous complications. The preoperative firsthand knowledge of the displacement of the vein and/or tributaries is very crucial in avoiding major venous complications. In cases of PCM, the vein is often displaced posteriorly by the tumor because the lesion originates anterior to the draining point of the SPV, whereas in cases of posterior petrous meningioma, the vein is usually displaced anteriorly. ${ }^{21}$ Preoperative CT digital subtraction venography or MR venography is a useful tool for assessing the petrosal vein and its tributaries in order to avoid surgical complications. ${ }^{19,27}$ The surgical technique described by Haq et al., which applies the combined petrosal approach without sectioning SPV, and the dural opening technique proposed in the same article are the other likely surgical nuances that can be used to avoid occlusion of the SPV.11

Elhammady and Heros hypothesized that there might be differences in sectioning the SPV based on the underlying pathology. A normal anatomy of SPV could be expected in MVD surgery, whereas the normal anatomy might get distorted in a pathological entity like petrous meningioma due to the encasement of the tributaries and the resultant damage to these vessels while achieving tumor decompression. So the investigators suggested that the obliteration of SPV in MVD surgery might be safe due to the intact compensatory venous outflow, whereas the procedure might turn out to be dangerous in tumor pathologies. ${ }^{9}$

The SPV should be considered a critical structure in posterior skull base approaches, and it has to be preserved in almost all cases. The preoperative angiographic evaluation of the SPV complex, including anatomical variations; the Ohata dural opening technique; early identification of the SPV and its diameter; avoidance of simultaneous and continuous cerebellar retraction; meticulous care of 
the dependent SPV stem segment in the case of severed tributaries; proximity to the brainstem while severing small-diameter tributaries; surgery for tumor pathology; endoscope-assisted surgery for better visualization; and good anatomical knowledge are the key factors that play a role in avoiding the complications caused by SPV complex occlusion. However, it may not be possible to preserve it in many situations, such as a tumor involving the petrous apex in which the SPV may be encased within the tumor, and the vein may have to be obliterated during the surgical procedure due to its proximity or adherence to the tumor. ${ }^{11}$ At times, SPV may be the offending vessel in a case of TN due to venous neurovascular conflict., ${ }^{77}$ Similarly, in situations in which the standard suboccipital approach is modified with a suprameatal or supratentorial approach, the preservation of SPV may be difficult..$^{33}$ The intraoperative transient obliteration of the vein of Dandy with simultaneous brainstem auditory or somatosensory evoked potential, or assessment of collateral venous drainage performed using indocyanine green prior to the cauterization of SPV in such situations, may help in predicting the occurrence of venous hypertension due to SPV occlusion in the postoperative period..$^{15,18,20}$

\section{Limitations of the Study}

The study has certain limitations. Because many of the literature series addressing the complications after sacrifice of the vein of Dandy are anecdotal case reports, the exact incidence of individual complications could not be assessed. Also, the literature search strategy was centered on the keywords "complications after SPV sacrifice"-and therefore many articles on CPA pathologies (especially the surgical treatment of TN) that did not focus on complications of Dandy's vein sacrifice or occlusion could not be included. Our article attempts to give an overview of the incidence and various patterns of complications after SPV sacrifice from the reported case series.

\section{Conclusions}

The SPV and its relation to the trigeminal nerve during the retrosigmoid approach were first described by Walter Dandy in 1929. Although Dr. Dandy described the obliteration of this vein as the second step in his surgery for $\mathrm{TN}$, he emphasized that it was required in only 1 in 15 cases. His concern may have been to prevent the torrential bleeding from this venous channel that may occur during cerebellar retraction, especially in the premicroscopic era when controlling such bleeding would have been an arduous task.

Currently, the preservation of the vein of Dandy is a neurosurgical dilemma. Literature review and experiences from large series suggest that obliterating the vein of Dandy while approaching the superior CPA corridor may be associated with negligible complications. However, the counterview cannot be neglected in light of some series showing up to a $30 \%$ complication rate from SPV sacrifice. It may be time to consider venous complications as we attempt to achieve a better safety profile in our surgical endeavors, and SPV sacrifice should be considered only in unavoidable situations. The intraoperative adjuncts such as brainstem evoked potential response and collateral flow assessment performed using indocyanine green angiography can be considered before the occlusion of SPV.

Venous complications have traditionally been neglected and attributed to other confounding variables, like retraction injury and peritumoral brain manipulation. This may be likely in the context of SPV sacrifice as well, and this review provides us the insight that although complications due to SPV obliteration are rare, they can happen, and the sequelae might be worse than the natural history of the existing pathology. Therefore, SPV preservation should be attempted whenever possible for the better safety profile of the patient and to optimize outcome.

\section{References}

1. Andeweg J: Consequences of the anatomy of deep venous outflow from the brain. Neuroradiology 41:233-241, 1999

2. Anichini G, Iqbal M, Rafiq NM, Ironside JW, Kamel M: Sacrificing the superior petrosal vein during microvascular decompression. Is it safe? Learning the hard way. Case report and review of literature. Surg Neurol Int 7 (Suppl 14):S415-S420, 2016

3. Chen HJ, Lui CC: Peduncular hallucinosis following microvascular decompression for trigeminal neuralgia: report of a case. J Formos Med Assoc 94:503-505, 1995

4. Cheng L: Complications after obliteration of the superior petrosal vein: Are they rare or just underreported? J Clin Neurosci 31:1-3, 2016

5. Dandy WE: An operation for the cure of tic douloureux: partial section of the sensory root at the pons. Arch Surg 18:687-734, 1929

6. Dandy WE: The treatment of trigeminal neuralgia by the cerebellar route. Ann Surg 96:787-795, 1932

7. Dumot C, Brinzeu A, Berthiller J, Sindou M: Trigeminal neuralgia due to venous neurovascular conflicts: outcome after microvascular decompression in a series of 55 consecutive patients. Acta Neurochir (Wien) 159:237-249, 2017

8. Dumot C, Sindou M: Trigeminal neuralgia due to neurovascular conflicts from venous origin: an anatomical-surgical study (consecutive series of 124 operated cases). Acta Neurochir (Wien) 157:455-466, 2015

9. Elhammady MS, Heros RC: Cerebral veins: to sacrifice or not to sacrifice, that is the question. World Neurosurg 83:320-324, 2015 A

10. Gharabaghi A, Koerbel A, Löwenheim H, Kaminsky J, Samii M, Tatagiba M: The impact of petrosal vein preservation on postoperative auditory function in surgery of petrous apex meningiomas. Neurosurgery 59 (1 Suppl 1):ONS68ONS74, 2006

11. Haq IBI, Susilo RI, Goto T, Ohata K: Dural incision in the petrosal approach with preservation of the superior petrosal vein. J Neurosurg 124:1074-1078, 2016

12. Huang YP, Wolf BS, Antin SP, Okudera T: The veins of the posterior fossa-anterior or petrosal draining group. Am J Roentgenol Radium Ther Nucl Med 104:36-56, 1968

13. Inamasu J, Shiobara R, Kawase T, Kanzaki J: Haemorrhagic venous infarction following the posterior petrosal approach for acoustic neurinoma surgery: a report of two cases. Eur Arch Otorhinolaryngol 259:162-165, 2002

14. Kaku S, Miyahara K, Fujitsu K, Hataoka S, Tanino S, Okada T, et al: Drainage pathway of the superior petrosal vein evaluated by CT venography in petroclival meningioma surgery. $\mathbf{J}$ Neurol Surg B Skull Base 73:316-320, 2012

15. Koerbel A, Gharabaghi A, Safavi-Abbasi S, Samii A, Ebner FH, Samii M, et al: Venous complications following petrosal vein sectioning in surgery of petrous apex meningiomas. Eur J Surg Oncol 35:773-779, 2009 
16. Koerbel A, Wolf SA, Kiss A: Peduncular hallucinosis after sacrifice of veins of the petrosal venous complex for trigeminal neuralgia. Acta Neurochir (Wien) 149:831-833, 2007

17. Kuncz A, Vörös E, Barzó P, Tajti J, Milassin P, Mucsi Z, et al: Comparison of clinical symptoms and magnetic resonance angiographic (MRA) results in patients with trigeminal neuralgia and persistent idiopathic facial pain. Medium-term outcome after microvascular decompression of cases with positive MRA findings. Cephalalgia 26:266-276, 2006

18. Liebelt BD, Barber SM, Desai VR, Harper R, Zhang J, Parrish R, et al: Superior petrosal vein sacrifice during microvascular decompression: perioperative complication rates and comparison with venous preservation. World Neurosurg 104:788-794, 2017

19. Masuoka J, Matsushima T, Hikita T, Inoue E: Cerebellar swelling after sacrifice of the superior petrosal vein during microvascular decompression for trigeminal neuralgia. J Clin Neurosci 16:1342-1344, 2009

20. Matsushima K, Ribas ES, Kiyosue H, Komune N, Miki K, Rhoton AL: Absence of the superior petrosal veins and sinus: surgical considerations. Surg Neurol Int 6:34, 2015

21. Matsushima T, Rhoton AL Jr, de Oliveira E, Peace D: Microsurgical anatomy of the veins of the posterior fossa. J Neurosurg 59:63-105, 1983

22. McLaughlin MR, Jannetta PJ, Clyde BL, Subach BR, Comey $\mathrm{CH}$, Resnick DK: Microvascular decompression of cranial nerves: lessons learned after 4400 operations. J Neurosurg 90:1-8, 1999

23. Mizutani K, Toda M, Yoshida K: The analysis of the petrosal vein to prevent venous complications during the anterior transpetrosal approach in the resection of petroclival meningioma. World Neurosurg 93:175-182, 2016

24. Pathmanaban ON, O'Brien F, Al-Tamimi YZ, HammerbeckWard CL, Rutherford SA, King AT: Safety of superior petrosal vein sacrifice during microvascular decompression of the trigeminal nerve. World Neurosurg 103:84-87, 2017

25. Perrini P, Di Russo P, Benedetto N: Fatal cerebellar infarction after sacrifice of the superior petrosal vein during surgery for petrosal apex meningioma. J Clin Neurosci 35:144-145, 2017

26. Ryu H, Yamamoto S, Sugiyama K, Yokota N, Tanaka T: Neurovascular decompression for trigeminal neuralgia in elderly patients. Neurol Med Chir (Tokyo) 39:226-230, 1999

27. Sakata K, Al-Mefty O, Yamamoto I: Venous consideration in petrosal approach: microsurgical anatomy of the temporal bridging vein. Neurosurgery 47:153-161, 2000

28. Samii M, Tatagiba M, Carvalho GA: Retrosigmoid intradural suprameatal approach to Meckel's cave and the middle fossa: surgical technique and outcome. J Neurosurg 92:235-241, 2000
29. Singh D, Jagetia A, Sinha S: Brain stem infarction: a complication of microvascular decompression for trigeminal neuralgia. Neurol India 54:325-326, 2006

30. Strauss C, Naraghi R, Bischoff B, Huk WJ, Romstöck J: Contralateral hearing loss as an effect of venous congestion at the ipsilateral inferior colliculus after microvascular decompression: report of a case. J Neurol Neurosurg Psychiatry 69:679-682, 2000

31. Tanriover N, Abe H, Rhoton AL Jr, Kawashima M, Sanus GZ, Akar Z: Microsurgical anatomy of the superior petrosal venous complex: new classifications and implications for subtemporal transtentorial and retrosigmoid suprameatal approaches. J Neurosurg 106:1041-1050, 2007

32. Tsukamoto H, Matsushima T, Fujiwara S, Fukui M: Peduncular hallucinosis following microvascular decompression for trigeminal neuralgia: case report. Surg Neurol 40:31-34, 1993

33. Watanabe T, Igarashi T, Fukushima T, Yoshino A, Katayama $Y$ : Anatomical variation of superior petrosal vein and its management during surgery for cerebellopontine angle meningiomas. Acta Neurochir (Wien) 155:1871-1878, 2013

34. Xi J, Ding X, Peng Z, Liu Q, Yuan X: [Protection of the superior petrosal vein in microneurosurgery for acoustic neuroma.] Zhong Nan Da Xue Xue Bao Yi Xue Ban 38:695-698, 2013 (Chinese)

35. Zhong J, Li ST, Xu SQ, Wan L, Wang X: Management of petrosal veins during microvascular decompression for trigeminal neuralgia. Neurol Res 30:697-700, 2008

\section{Disclosures}

The authors report no conflict of interest concerning the materials or methods used in this study or the findings specified in this paper.

\section{Author Contributions}

Conception and design: Nanda, Narayan, Savardekar. Acquisition of data: Narayan, Savardekar, Patra, Thakur, Riaz. Analysis and interpretation of data: Nanda, Narayan, Savardekar. Drafting the article: Narayan, Savardekar. Critically revising the article: all authors. Reviewed submitted version of manuscript: Nanda, Patra, Mohammed, Thakur, Riaz. Approved the final version of the manuscript on behalf of all authors: Nanda. Administrative/technical/material support: Nanda. Study supervision: Nanda.

\section{Correspondence}

Anil Nanda: Louisiana State University Health Sciences Center, Shreveport, LA. ananda@1suhsc.edu. 